\title{
Osteonecrosis of the Jaw
}

\section{By Catherine Van Poznak, MD}

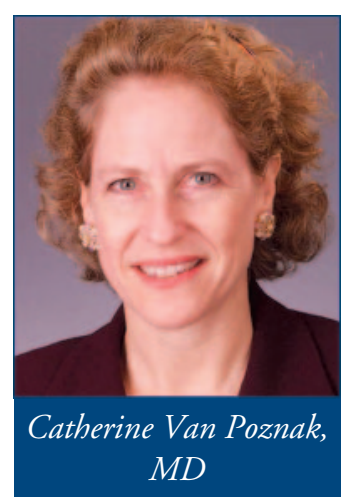

With broadened and prolonged use of bisphosphonates for advanced cancer becoming more common, osteonecrosis of the jaw (ONJ) has become a concern. We know little about the pathogenesis of ONJ, we cannot reliably predict who is at greatest risk for its development, and we cannot control the condition easily once it is diagnosed. These unknowns make ONJ worrisome to patients, clinicians, and those in the pharmaceutical

field. The article by Ruggiero et al, ${ }^{1}$ published in this issue of the Journal of Oncology Practice, sheds some light on ONJ by outlining what is and is not understood about the condition.

The authors show us that little is known about ONJ, and the condition currently lacks a consensus definition. Yet they manage to suggest useful guidelines for specific populations: patients with cancer who are initiating bisphosphonate therapy, those who are receiving bisphosphonate therapy, and those with established ONJ. Since 1994, ASCO has assembled expert panels to develop evidence-based oncology practice guidelines on a number of topics including the use of bisphosphonates in patients with breast cancer ${ }^{2}$ and multiple myeloma, ${ }^{3}$ but the Ruggiero guidelines were not developed through the ASCO mechanism.

Although ONJ is not seen commonly-incidence is estimated at approximately $1 \%$ to $10 \% 0^{4-6}$ in patients receiving intravenous bisphosphonates-it is likely that over time most clinicians will care for patients with this diagnosis, making awareness of it critically important. Hence, the article by Ruggiero et al makes a timely contribution. However, these guidelines are constrained by the sparse data available on ONJ, which consist of case reports or case series. This level of evidence would be considered of poor quality by the U.S. Preventive Services Task Force grading scheme, ${ }^{7}$ thereby restricting the scientific rigor with which guidelines can be drawn. Nevertheless, the Ruggiero article reflects the reality that we do need to manage patients now in advance of adequate data and a complete understanding of the disease process; the report serves as an ONJ reference for managing patients with cancer being treated with bisphosphonate therapy.

The importance of maintaining good oral hygiene is well recognized, and patients with cancer may be at increased risk for compromised oral health. The American Dental Association $^{8}$ and the National Institutes of Health ${ }^{9}$ have generated recommendations for the dental care of patients with cancer. These include a thorough dental evaluation prior to beginning therapy, and maximizing oral health with a wellbalanced diet, avoidance of tobacco and alcohol, the use of mouth rinses, and follow-up dental care. Encouraging oral health is sound advice and can be applied easily to those initiating bisphosphonate therapy as well, which Ruggiero et al recommend. However, there are no data demonstrating that such oral health practices alter the risk or the course of ONJ.

Clearly, we need to better define ONJ, including specifically its pathogenesis and optimal management. If there is an association between bisphosphonate therapy and ONJ, then ONJ could be expected to become more prevalent as the use of bisphosphonates expands for both malignant and nonmalignant conditions. Prospective clinical trials that will explore ONJ include Novartis study 2352, investigating the long term use of zoledronic acid in patients with metastatic breast cancer, and the Southwest Oncology Group 0307 randomized study of adjuvant bisphosphonate therapy in breast cancer patients. Novel agents targeting bone are currently being developed, and may offer a different risk profile; examples include denosumab (AMG 162, monoclonal antibody to NF- $\kappa$ B ligand) and inhibitors of cathepsin. To increase the base of our knowledge, cases of confirmed or suspected ONJ should be reported to the Federal Drug Administration through Medwatch. ${ }^{10}$

Every advance in the treatment of cancer is associated with some change in risk. Certainly the bisphosphonate experience fits this rule. ONJ, as possibly associated with bisphosphonate use, is increasingly affecting the lives of patients with cancer and warrants attention. The guidelines in this issue of $J O P^{1}$ may help clinicians address the issues concerning ONJ. They serve as a step in setting practice standards. As additional data are obtained and our depth of knowledge on ONJ expands, we can look forward to updated evidence-based guidelines.

Catherine Van Poznak, MD, is a medical oncologist at Memorial Sloan-Kettering Cancer Clinic, New York, New York.

\section{References}

1. Ruggiero S, Gralow J, Marx R, et al: Practical guidelines for the prevention, diagnosis, and treatment of osteonecrosis of the jaw in patients with cancer. J Oncol Pract 2:7-14, 2006

2. Hillner BE, Ingle JN, Chlebowski RT, et al: American Society of Clinical
Oncology 2003 update on the role of bisphosphonates and bone health issues in women with breast cancer. J Clin Oncol 21:4042-4057, 2003.

3. Berenson JR, Hillner BE, Kyle RA, et al: American Society of Clinical 
Oncology clinical practice guidelines: The role of bisphosphonates in multiple myeloma. J Clin Oncol 20:3719-3736, 2002

4. Van Poznak CH, Estilo CL, Sauter NP, et al: Osteonecrosis of the jaw in patients with metastatic breast cancer. Breast Cancer Res Treat 88:S131, 2004 (suppl 1; abstr 3057)

5. Hoff $\mathrm{AO}$, Toth $\mathrm{B}$, Altundag $\mathrm{K}$, et al: Osteonecrosis of the jaw in patients receiving intravenous bisphosphonate therapy. J Bone Min Res 20:S55, 2005 (abstr 1218)

6. Durie BGM, Katz M, Crowley J: Osteonecrosis of the jaw and bisphosphonates. N Engl J Med 353:99, 2005
7. US Preventive Services Task Force Ratings: Strength of recommendations and quality of evidence. http://www.ahrq.gov/clinic/3rduspstf/ratings.htm

8. For the dental patient: Oral care for cancer patients. J Am Dent Assoc 133: 1014, 2002

9. National Institutes of Health: Oral complications of chemotherapy and head/ neck radiation. http://www.cancer.gov/cancertopics/pdq/supportivecare/ oralcomplications/HealthProfessional

10. United States Food and Drug Administration: MedWatch reporting forms 2005. http://www.fda.gov/medwatch/getforms.htm

\section{ASCO/AAMC Oncologist Workforce Survey}

You may be selected to participate in an important oncologist workforce survey being conducted by ASCO and the AAMC Center for Workforce Studies. Results from the study will help determine future oncology workforce needs and inform planning. The survey includes questions on:

- Your practice and patient volume

- Job satisfaction

- Feelings of burnout

- Use of nurse practitioners and physician assistants

- Views on approaches to assuring future access to oncology care

The survey will be administered in January 2006, and summary results will be reported at the 2006 ASCO Annual Meeting.

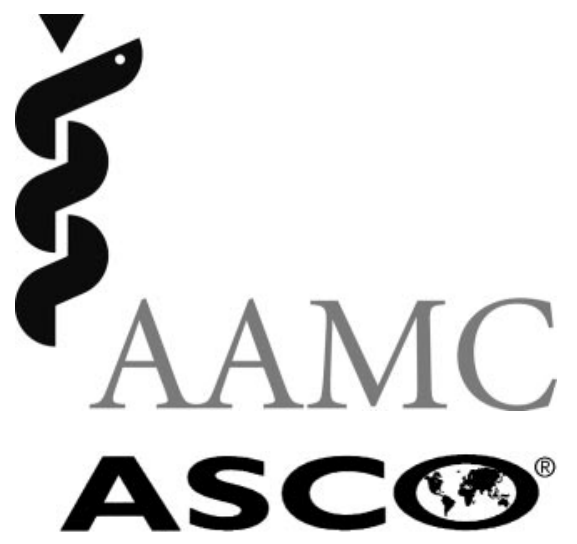

